\title{
O ensino de cálculo diferencial e integral sob a óptica da teoria dos campos conceituais
}

\author{
Tailon Thiele*, Eliane Miotto Kamphorst ${ }^{\star \star}$, Carmo Henrique Kamphorst***
}

\section{Resumo}

A Teoria dos Campos Conceituais é uma teoria psicológica, fruto de um estudo realizado por Gèrard Vergnaud, embasado nas ideias de Piaget e Vigotsky. Esta pesquisa tem como objetivos o entendimento dessa teoria, além da contextualização com o ensino de Cálculo Diferencial e Integral. A partir de uma pesquisa bibliográfica, foram descritos os principais pontos da teoria dos Campos Conceituais, com destaque para o tripleto (S, I, R), o qual representa as situações, os invariantes operatórios e as representações, respectivamente. A compreensão desses elementos, de acordo com a teoria de Vergnaud, é imprescindível para que o docente possa entender os processos cognitivos dos aprendizes e, consequentemente, intervir na aprendizagem. Quanto ao ensino do Cálculo Diferencial e Integral, é possível perceber que os invariantes operatórios têm importância singular na aprendizagem dos conceitos. Assim, o docente precisa propor aos discentes situações que elucidem de maneira correta e coerente os conceitos. De maneira geral, foi possível compreender a teoria dos Campos Conceituais e construir um embasamento que pode contribuir com a amenização das dificuldades na aprendizagem do Cálculo, além de possibilitar a continuidade dos estudos nesse viés.

Palavras-chave: Campos Conceituais. Ensino. Cálculo Diferencial e Integral.

\section{Introdução}

De acordo com Lima e Silva (2015),

Gerard Vergnaud, em sua teoria dos Campos Conceituais, traz contribuições no contexto da reflexão sobre aprendizagem e desenvolvimento, com conexões evidentes com as ideias de Piaget e Vigotsky, que são acrescentadas com contribuições específica e original, o que este autor denomina "Teoria da Referência". O que a Teoria da Referência propõe é a conexão necessária dos conceitos a um domínio epistemológico específico [...] (p. 59).

Assim, a Teoria dos Campos Conceituais tem como proposta uma grande contribuição no processo de ensino e aprendizagem, principalmente no que

* Universidade Regional Integrada do Alto Uruguai e das Missões. E-mail: thiele.tailon@gmail.com

** Universidade Regional Integrada do Alto Uruguai e das Missões. E-mail: anne@uri.edu.br

*** Universidade Regional Integrada do Alto Uruguai e das Missões. E-mail: carmo@uri.edu.br

Recebido em: 01/10/2018 - Aceito em: 15/10/2018.

https://doi.org/10.5335/rbecm.v1i2.8976 
tange à compreensão dos processos cognitivos dos aprendizes. Isso é possível em função de que, de acordo com a teoria, a conceitualização é o ponto chave para o desenvolvimento cognitivo. Dessa maneira, por se tratar de um estudo psicológico, esse processo de conceitualização pode ser observado no sentido de identificar rupturas que se constituem como barreiras para a aprendizagem.

Os estudos de Vergnaud podem contribuir de maneira significativa no ensino e aprendizagem do Cálculo Diferencial e Integral, visto que essa é uma disciplina que desperta receio em boa parte dos alunos de graduação e que possui altos índices de reprovação e desistência. Diante desse cenário, justifica-se a importância de estudos que busquem diminuir as dificuldades encontradas na aprendizagem dos conceitos dessa disciplina.

Dessa forma, este estudo busca realizar uma pesquisa bibliográfica acerca da Teoria dos Campos Conceituais, a fim de compreender as ideias de Vergnaud no que se refere à aprendizagem por meio da conceitualização. Posteriormente, almeja-se contextualizar essas ideias no ensino do Cálculo Diferencial e Integral, no sentido de contribuir com o entendimento dos processos cognitivos que fazem parte da aprendizagem dos conceitos dessa disciplina.

\section{A Teoria dos Campos Conceituais de Vergnaud}

Gèrard Vergnaud, autor da Teoria dos Campos Conceituais, é um psicólogo seguidor dos estudos de Piaget. Ambos procuram analisar o sujeito do conhecimento a partir de uma resposta a uma situação. Segundo Carvalho Jr. e Aguiar Jr. (2008), as investigações de Piaget e Vergnaud se complementam no sentido da intervenção didática em sala de aula.

Visto que Vergnaud leva em consideração o sujeito em situação, é possível pensar em situações didáticas que tomem como referência as características dos conteúdos estudados em sala de aula. Segundo o autor, o conteúdo tem importância significativa no processo de aprendizagem, uma vez que a conceitualização do real não se trata de um processo simples, mas uma atividade psicológica.

Moreira (2002) apresenta uma definição ampla da teoria dos Campos Conceituais, proposta por Gèrard Vergnaud. Segundo ele, essa teoria pode ser entendida como um

[...] conjunto informal e heterogêneo de problemas, situações, conceitos, relações, estruturas, conteúdos e operações de pensamento, conectados uns aos outros e, provavelmente, entrelaçados durante o processo de aquisição (MOREIRA, 2002, p. 7).

Pais (2002) entende que uma das propostas da teoria dos Campos Con- 
ceituais trata de rever as condições da aprendizagem conceitual, a fim de que essa se torne possível no que se refere à compreensão do aluno. É o estudo do âmbito do significado dos conceitos no contexto escolar, sem desligar-se de suas raízes epistemológicas e fornece uma referência semelhante à complexidade do fenômeno da aprendizagem.

Ainda de acordo com o mesmo autor, a teoria dos campos conceituais foi desenvolvida na perspectiva da análise do entendimento do significado do saber escolar pelo aluno. Segundo ele,

[...] o conhecimento passa a ser concebido como uma sucessão de adaptações que o aluno realiza sob a influência de situações que ele vivencia na escola e na vida cotidiana [...] (PAIS, 2002, p. 53).

Isso explica a concepção de Vergnaud acerca do sujeito em situação, na qual o aluno compreende o conteúdo de acordo com as diversas situações em que é exposto.

Além disso, o processo de aprendizagem descrito nessa teoria destaca a utilização de conhecimentos anteriores e a capacidade de sintetizar e contextualizar essas informações aos novos conhecimentos. Essa ideia é evidenciada por Pais,

No caso ideal em que a aprendizagem acontece com sucesso, os conhecimentos anteriores são adicionados uns aos outros e incorporados à nova situação. Assim, ocorre uma parte do processo cognitivo que consiste no conjunto de procedimentos de raciocínio desenvolvidos pelo sujeito para coordenar as adaptações necessárias para que informações precedentes sejam incorporadas em uma situação de aprendizagem, sintetizando o novo conhecimento (PAIS, 2002, p. 53).

Essa teoria proposta por Vergnaud é uma teoria cognitivista, a qual busca estudar o desenvolvimento e a aprendizagem de competências complexas, fornecendo alguns princípios básicos atribuídos a esses processos. Franchi (1999) afirma que um dos pressupostos básicos dos Campos Conceituais considera que o conhecimento se constitui e se desenvolve ao longo do tempo através da interação adaptativa do aluno com as suas situações experiências. Segundo ela, o funcionamento cognitivo do indivíduo em situação, debruça-se sobre os conhecimentos já construídos e, mutuamente, o indivíduo incorpora novos aspectos a esses conhecimentos, construindo competências progressivamente mais complexas.

Ainda é importante salientar que, na perspectiva da teoria dos Campos Conceituais, os processos cognitivos são compreendidos como a organização da conduta, a representação, e a percepção, bem como a evolução de competências e de concepções de um indivíduo ao longo de sua experiência.

Para exemplificar os Campos Conceituais, Moreira (2002) cita os campos conceituais das estruturas aditivas, as quais envolvem a adição ou a subtração, 
ou ambas combinadas. Já o campo conceitual das estruturas multiplicativas, exigem o domínio da multiplicação ou da divisão, ou uma combinação entre as elas. Entretanto, o autor destaca que Vergnaud considera que os diferentes campos conceituais não são independentes. Segundo ele, é preciso fazer alguns recortes, porém é praticamente impossível estudar as coisas separadamente.

Portanto, é possível compreender essa teoria como um conjunto de conceitos, conforme evidenciam Silva e Lehmann (2012):

Entendemos campo conceitual como um conjunto de conceitos. Para que os conceitos sejam construídos por parte dos sujeitos, diferentes situações devem ser apresentadas, e para Vergnaud, o conceito de situação está relacionado à tarefa, sendo que toda situação deve ser analisada como um conjunto de tarefas. Quando uma situação é apresentada ao aluno, observamos que cada um age e responde de acordo com suas próprias representações, criando esquemas de pensamento diferenciados (SILVA; LEHMANN, 2012, p. 69).

Nesse sentido, é necessário entender as definições de conceito, situação e esquema, sob o olhar da teoria dos Campos Conceituais.

\section{Conceito}

Pais (2002) considera que os conceitos são concepções gerais e abstratas desenvolvidas em uma área específica de conhecimento, formadas para sintetizar a essência de uma classe de objetos, situações ou problemas que possuem relação com o mundo da vida. Além disso, o autor ressalta a importância da aprendizagem de conceitos. Segundo ele, existe uma tendência na prática de ensino da matemática que enaltece a memorização de fórmulas, regras, definições, teoremas e demonstrações. Isso favorece mais a reprodução de modelos do que a aprendizagem dos conceitos. Pais considera que essa concepção de educação não supre as exigências da sociedade tecnológica, e que, assim, deve-se abrir um espaço maior para o estudo da formação dos conceitos.

De acordo com Moreira (2002) conceito, para Vergnaud é definido como um tripleto de três conjuntos, quais sejam $\mathrm{S}$, I, R. Segundo o autor,

S é um conjunto de situações que dão sentido ao conceito;

I é um conjunto de invariantes (objetos, propriedades e relações) sobre os quais repousa a operacionalidade do conceito, ou o conjunto de invariantes operatórios associados ao conceito, ou o conjunto de invariantes que podem ser reconhecidos e usados pelos sujeitos para analisar e dominar as situações do primeiro conjunto; $R$ é um conjunto de representações simbólicas (linguagem natural, gráficos e diagramas, sentenças formais, etc.) que podem ser usadas para indicar e representar esses invariantes e, conseqüentemente, representar as situações e os procedimentos para lidar com elas (MOREIRA, 2002, p. 10). 
Dessa maneira, o primeiro conjunto (S) trata das situações, porta de entrada para o estudo do sujeito em situação, de Vergnaud e que dão sentido ao conceito. O Segundo conjunto (I) são os invariantes operatórios que podem ser utilizados pelo indivíduo para resolver as situações, ou seja, o significado do conceito. Enquanto isso, o terceiro conjunto $(\mathrm{R})$ refere-se às representações simbólicas que servem de base para indicar e representar os invariantes, ou seja, o significante.

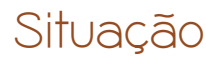

Moreira (2002) esclarece que, para Vergnaud, o conceito de situação é o mesmo de situação didática. Segundo o autor, situação se refere à tarefa, e uma situação complexa pode ser considerada como uma combinação de tarefas, para as quais se deve estar ciente de suas naturezas e dificuldades próprias. O mesmo autor descreve que Vergnaud

[...] destaca duas ideias principais em relação ao sentido de situação: variedade e história. Isto é, em um certo campo conceitual existe uma grande variedade de situações e os conhecimentos dos alunos são moldados pelas situações que encontram e progressivamente dominam, particularmente pelas primeiras situações suscetíveis de dar sentido aos conceitos e procedimentos que queremos que aprendam (ibid.). Segundo Vergnaud, muitas de nossas concepções vêm das primeiras situações que fomos capazes de dominar ou de nossa experiência tentando modificá-las (MOREIRA, 2002, p. 11).
Como já foi destacado anteriormente, são as situações que dão sentido ao conceito, considerando o tripleto $\mathrm{S}, \mathrm{I}, \mathrm{R}$, e que servem como base para o estudo do sujeito em ação, foco de Vergnaud. Carvalho Jr. e Aguiar Jr. $(2008)^{1}$ descrevem uma situação como sendo os problemas que o indivíduo precisa resolver.

Magina (2005) diz que as competências e concepções do sujeito vão se aprimorando ao longo do tempo, a partir da experiência com diversas situações dentro e fora da escola. Segundo ela, os alunos utilizam os conhecimentos construídos em situações anteriores para adaptá-los a novas situações que venham a surgir. Assim, é possível dizer que o conhecimento acontece a partir do momento em que o aluno entra em contato com situações as quais já possui familiaridade.

De acordo com Lima e Santos (2015), as situações são divididas em duas classes, as quais são descritas a seguir:

Primeira classe de situações: 0 sujeito já possui em seu repertório de competências os procedimentos adequados ao tratamento da situação. Trata-se mais de uma relação de filiação aos conhecimentos pré-existentes;

Segunda classe de situações: o sujeito não dispõe de todas as competências requeridas para o tratamento da situação. Há uma ruptura do conhecimento e um momento de descoberta e, em alguns casos, de invenção do novo. Para a resolução da nova situação ou problema (considerada como nova pelo aprendiz) são necessárias reflexão e exploração, que podem conduzir ao sucesso ou ao fracasso (LIMA; SANTOS, 2015, p. 60 - 61). 
A seguir, será descrito o conceito de esquema, no que se refere à teoria dos Campos Conceituais, conceito que possui estreita relação com as situações.

\section{Esquema}

Moreira (2002) afirma que, para Vergnaud, esquema se trata da organização invariante acerca do comportamento de uma classe de situações. Segundo ele, a partir dos esquemas é que devem ser pesquisados os conhecimentos-em-ação do indivíduo, ou seja, os elementos cognitivos que contribuem para que a ação desse indivíduo seja operatória.

De acordo com o mesmo autor,

Esquema é o conceito introduzido por Piaget para dar conta das formas de organização tanto das habilidades sensório-motoras como das habilidades intelectuais. Um esquema gera ações e deve conter regras, mas não é um estereótipo porque a sequência de ações depende dos parâmetros da situação (1994, p. 53). Um esquema é um universal que é eficiente para toda uma gama de situações e pode gerar diferentes sequências de ação, de coleta de informações e de controle, dependendo das características de cada situação particular. Não é o comportamento que é invariante, mas a organização do comportamento (MOREIRA, 2002, p. 12).

Em resumo, o conceito de esquema pode ser entendido como a organização invariante do modo como se comporta uma determinada classe de situação. Lima e Santos (2015) destacam a importância do papel do professor no que se refere às situações e esquemas. Segundo os autores, o docente é responsável por oferecer aos alunos situações que aumentem o repertório de esquemas, auxiliando no desenvolvimento cognitivo dos discentes.

É importante destacar aqui os invariantes operatórios (conceito-em-ação e teorema-em-ação). De acordo com Moreira (2002), visto que o esquema se trata da organização de uma classe de situações, os invariantes operatórios são elementos importantes dos esquemas. Segundo ele,

Teorema-em-ação é uma proposição tida como verdadeira sobre o real. Conceito-em-ação é um objeto, um predicado, ou uma categoria de pensamento tida como pertinente, relevante (MOREIRA, 2002, p. 14).

\section{Justificativas para a Teoria dos Campos Conceituais}

Lima e Santos (2015), referindo-se ao tripleto $(\mathrm{S}, \mathrm{I}, \mathrm{R})$, descrevem de maneira sucinta os três principais argumentos que serviram de base para Vergnaud chegar ao conceito de Campo Conceitual. São eles:

- Um conceito não se forma dentro de um único tipo de situação e sim de um conjunto delas. Para formar um conceito é necessário o isomorfismo e a indução, além de hermenêuticas de forma e/ou conteúdo entre situações; 
- Uma situação não se analisa com um único conceito e sim com um conjunto híbrido de conceitos;

- A construção e a apropriação de todas as propriedades de um conceito ou de todos os aspectos de uma situação é um processo de muito fôlego que se desenrola ao longo dos anos, às vezes uma dezena de anos, com analogias e mal-entendidos entre situações, entre concepções, entre procedimentos, entre significantes. Até que o conceito é por fim estabelecido (LIMA; SANTOS, 2015, p. 91-92).

Então, um conceito apenas torna-se significativo para o discente, quando é relacionado com um leque de situações e aspectos nos quais ele está envolvido. Simultaneamente, uma situação deve ser analisada a partir de vários conceitos. Dessa forma, o processo de aprendizagem torna-se longo, até que as ideias de um campo conceitual tornam-se totalmente esclarecidas.

\section{A Teoria dos Campos Conceituais e o ensino de Cálculo Diferencial e Integral}

Lima e Santos (2015) esclarecem que a Teoria dos Campos Conceituais, estudada por Gèrard Vergnaud, busca entender e explicar o desenvolvimento dos processos de conceitualização, a par- tir do pressuposto de que grande parte dos conhecimentos se forma por competências que fazem parte do repertório de esquemas do aluno.

Esses mesmos autores, realizaram um estudo da teoria de Vergnaud sob uma óptica voltada ao ensino da disciplina de Cálculo, em cursos superiores não voltados à formação de matemáticos. Eles constataram que um dos elementos mais importantes no que se refere à teoria dos Campos Conceituais aplicada ao ensino de Cálculo são os invariantes operatórios apresentados pelos alunos. Segundo eles, a construção e formação de um conceito dependem do tripleto (S, I, R), entretanto destacam-se os invariantes operatórios como principal fator, tanto no viés de formação quanto na forma que são trabalhados.

Quanto aos conceitos ensinados na disciplina de Cálculo, destacam-se as funções, limites e derivadas. Dessa forma, como explanado anteriormente, o docente deve observar os invariantes operatórios encontrados pelos discentes. Se esses invariantes correspondem com o sentido que o conceito estudado exige, o professor pode prosseguir. Entretanto, se esses invariantes não condizem com o almejado, é necessário tomar um novo direcionamento para as aulas. Isso pode ser evidenciado, segundo Lima et al. (2017). Segundo eles, quando 
[...] o aluno construiu um campo conceitual particular referente aos limites, as derivadas e as funções, de forma que seus invariantes permeiem os sentidos exatos (aproximação, taxa de variação e correspondência, respectivamente), então a estrutura de direcionamento das aulas pode permanecer. Caso contrário, o professor deve intervir para linearizar as rupturas existentes na formação dos conceitos (LIMA et al., 2017, p. 4).

Um problema enfrentado nessa disciplina é que, muitas vezes, os problemas não são absorvidos e/ou resolvidos pelos estudantes, em função de não haver, em alguns casos, um conjunto de situações que os alunos consigam entender. Por isso, Lima e Silva propõem que o docente pode elucidar o invariante operatório por meio de situações que facilitem esse processo. Isso deve ser feito com o maior número possível de exemplos, os quais vão permitir que o aluno amplie seu entendimento, explorando um leque maior de interpretações que um conceito pode ter.

Em qualquer campo conceitual, espera-se que o aluno consiga aplicar um conceito em diferentes situações, processo chamado, por Vergnaud, de "capacidade de transferência". No caso da disciplina de Cálculo Diferencial e Integral, os alunos terão adquirido os conceitos ensinados na disciplina, se:

- Relacionam os conceitos de limite ao de derivada;

- Definem as etapas de construção de forma coerente, seja na forma escrita ou falada;
- Efetuam transferências em situações fora do contexto;

- Efetuam transferências em situações novas (concretas ou abstratas) (LIMA; SANTOS, 2015, p. 102).

Lehmann e Silva (2012) também realizaram um estudo que aborda a teoria dos Campos Conceituais sob a perspectiva do ensino do cálculo. Segundo esses autores, o desenvolvimento cognitivo do aluno passa pela conceitualização. Esse processo depende das situações levantadas pelo professor, as quais vão determinar os invariantes operatórios de cada aluno, já que a aprendizagem acontece individualmente e cada um pode construir diferentes esquemas. Assim, as situações devem promover reflexões e discussões entre os alunos, oferecendo assim, condições para que o professor analise os conceitos-em-ação e conceitos-em-ato manifestados durante esses momentos e defina a direção que a aula deve tomar.

Lima et al. (2017) fazem referência às representações (que fazem parte do tripleto S, I, R). Segundos esses autores, a construção dos invariantes operatórios está associada principalmente com as situações apresentadas pelo docente (como já descrito anteriormente), mas que muitas vezes, em Cálculo Diferencial, a notação pode trazer obstáculos para a aprendizagem. Isso ocorre em função de comumente a linguagem matemática do Cálculo acaba provocando impactos nos alunos. Por isso, as representações têm um importante pa- 
pel nos processos cognitivos, uma vez que devem ser coerentes e bem elaboradas, de acordo com o conceito estudado.

\section{Resultados e Discussões}

A teoria dos Campos Conceituais de Vergnaud possui ampla importância para o entendimento dos processos cognitivos dos aprendizes. Isso acontece devido ao fato de que essa teoria permite observar e entender separadamente cada fator que influencia na aprendizagem, tais como situações-problema, invariantes operatórios que darão origem aos esquemas e a própria representação que é dada a um conceito estudado. A partir do estudo desses fatores, representados pelo tripleto (S, I, R), é que o docente identifica as lacunas que persistem durante o processo de aprendizagem e as formas que pode vir a intervir para que haja a aprendizagem significativa.

No que se refere à disciplina de Cálculo Diferencial e Integral, estudos (LIMA; SANTOS, 2015; LIMA et al., 2017), apontam que os principais fatores que determinam a aprendizagem significativa dos conceitos são os conceitos-em-ação e conceitos-em-ato, também denominados de invariantes operatórios. Entretanto, a construção de invariantes operatórios coerentes com os conceitos estudados passa pela necessidade de haver situações que elucidem de maneira correta tais conceitos.
Ainda existem poucos estudos que relacionam a teoria dos Campos Conceituais ao ensino da disciplina de Cálculo, entretanto, os estudos que já existem corroboram entre si na maioria dos resultados. Todos eles levam o enfoque principal aos invariantes operatórios apresentados pelos sujeitos (discentes) durante as reflexões e as discussões propiciadas pelas situações propostas pelo docente. Observar esses invariantes conduz o professor a decidir se a aprendizagem condiz com o esperado, ou se a aula precisa de um redirecionamento.

Os alunos da disciplina de cálculo precisam, necessariamente, realizar conexões entre os conceitos estudados, evidenciando assim que houve uma aprendizagem significativa. A conceitualização das funções, limites e derivadas, devem proporcionar a possibilidade de relacionar esses conceitos entre si e aplicá-los em situações diversas, diferentes das estudadas até então.

\section{Conclusões e perspectivas}

- A teoria dos Campos Conceituais torna-se importante no ensino de cálculo, principalmente pela possibilidade de identificar as lacunas existentes nos processos cognitivos dos alunos;

- Os invariantes operatórios apresentados pelos alunos podem servir de aporte para o docente identificar 
essas lacunas na aprendizagem dos conceitos de funções, limites e derivadas;

- Para que aconteça a aprendizagem significativa dos conceitos de cálculo, o discente precisa ser capaz de relacionar esses conceitos entre si, além de aplicá-los em diferentes situações (daí a importância de o docente apresentar diversas situações);

- Ainda existem poucos estudos sobre essa teoria, principalmente no que se refere à aplicação ao Cálculo Diferencial e Integral. Isso nos remete à necessidade de novas pesquisas nesse viés. Durante a continuidade desta pesquisa, pretende-se construir um campo conceitual para o cálculo, apresentando o tripleto $(\mathrm{S}$, I, R) que o compõem.

\section{The teaching of differential} and integral calculus under the conceptual fields perspective Abstract

The Theory of Conceptual Fields is a psychological theory, result of a study done by Gèrard Vergnaud, based on Piaget and Vigotsky's ideas. This research aims to understand this theory and propose a link to the teaching of Differential and Integral Calculus. Through a bibliographic research the main points of Conceptual Fields were described, emphasizing the triplet (S, I, $R$ ), which represents the situations, the operative invariants and the representations, respectively. The understanding of these elements, according to Vergnaud, is indispensable for that the teacher could understand the cognitive processes of the learners and, consequently, step in on the leaning. Concerning the teaching of Differential and Integral Calculus, is possible to realize that the operative invariants have a singular importance on the learning of the concepts. Thus, the teacher has to offer ways of clarifying correct and consistently the concepts to the students. In general, it was possible to understand the Theory of Conceptual Fields and to raise concern that can contribute to the decreasing of difficulties related to the learning of Calculus, as well as to improve the studies in this perspective.

Keywords: Conceptual Fields. Teaching. Differential and Integral Calculus.

\section{Nota}

1 Os autores realizam um estudo baseado na teoria dos Campos Conceituais, com os conceitos de calor e temperatura, abordando o tripleto de cada um desses conceitos.

\section{Referências}

CARVALHO Jr, Gabriel Dias de; AGUIAR Jr, Orlando. Os Campos Conceituais de Vergnaud como ferramenta para o planejamento didático. Caderno Brasileiro de Ensino de Física, v. 25, n. 2, p. 207-227, ago. 2008.

FRANCHI, Anna. Considerações sobre a teoria dos Campos Conceituais. In: ALCÂNTARA, Sílvia Dias de et al. Educação Matemática: uma introdução. São Paulo: EDUC, 1999. p. 155-196.

LEHMANN, Monique Siqueira; SILVA, Júlio Cézar da. Análise da Contribuição da Teoria dos Campos Conceituais no Ensino de Cálculo em Cursos de Tecnologia. Revista Publicação técnico-científica do IST-Rio, n. 5, dez. 2012. 
LIMA, M. S.; SANTOS, J. V. C.; CAMPO, T. M. M. O Campo Conceitual do Cálculo Diferencial Sob o Olhar de Professores. In: COLÓQUIO INTERNACIONAL SOBRE A TEORIA DOS CAMPOS CONCEITUAIS, 2, 2017, Porto Alegre. Anais... Porto Alegre: GEEMPA, 2017. Disponível em: <http://www.geempa.com.br/wp-content/uploads/2017/08/O-Campo-Conceitual-do-C\%C3\%A1lculo-Diferencial-sob-o-Olhar-de-Professores.pdf.>. Acesso em: 02 jan. 2018.

LIMA, Melina Silva de; SANTOS, José Vicente Cardoso. A teoria dos campos conceituais e o ensino de cálculo. Curitiba: Appris, 2015.

MAGINA, Sandra. A Teoria dos Campos Conceituais: contribuições da Psicologia para a prática docente. In: ENCONTRO REGIONAL DE PROFESSORES DE MATEMÁTICA, 18, 2005. Campinas, SP. Anais... Campinas: Unicamp, 2005.

MOREIRA, Marco Antônio. A Teoria dos Campos Conceituais de Vergnaud, o Ensino de Ciências e a Pesquisa nesta Área. Investigações em Ensino de Ciências, v. 7, n. 1, p. 7-29, 2002.

PAIS, Luiz Carlos. Didática da Matemática: uma análise da influência francesa. 2. ed. Belo Horizonte: Autêntica, 2002, p. 51-64. 